\title{
La salud pública en las facultades de medicina y escuelas de enfermería de Cataluña. La visión del alumnado
}

\author{
N. Obradors-Rial ${ }^{a}$, A. Segura-Benedicto ${ }^{b}$
}

Introducción. La influencia de la formación universitaria sobre el futuro ejercicio profesional de la salud pública (SP) se desconoce. Dada la marginalidad de la SP en el sistema sanitario, se pretende conocer el peso del área de conocimiento en las titulaciones de ciencias de la salud y la percepción de los estudiantes de los últimos cursos de medicina y de enfermería sobre la SP. Sujetos y métodos. El estudio consta de dos partes: la descripción de la oferta formativa de asignaturas del área de conocimiento de la SP y la valoración que merecen estas asignaturas a una muestra de 361 estudiantes de medicina y de enfermería, mediante un cuestionario, objeto de análisis univariado. Resultados. Diez de las doce titulaciones de ciencias de la salud impartidas en nueve universidades catalanas ofrecen asignaturas del área de conocimiento con un peso entre 3 (CTA UB) y 32,5 (enfermería comunitaria UdL) créditos. Los estudiantes no tienen una clara percepción de la naturaleza de la SP. No obstante, la valoración de los estudiantes de enfermería es sistemáticamente mejor que la de los de medicina. La SP no despierta particulares expectativas de dedicación profesional, ni especial interés entre los estudiantes de medicina. Conclusión. Si bien el estudio se fundamenta en una primera aproximación, limitada a una muestra de conveniencia, y con toda la prudencia en la interpretación de los resultados, se reafirma la hipótesis de que las asignaturas del área de conocimiento de SP no estimulan a los estudiantes a plantearse en el futuro el ejercicio profesional de la SP.

Palabras clave. Estudiantes de enfermería. Estudiantes de medicina. Salud pública. Universidades.

\section{The public health in the universities of medicine and} schools of nursing in Catalonia. The students view

Introduction. The influence that the education in universities can have on the future of the professional practice in public health (PH) it's unknown. Because of the peripheral position of public health in the health system, this study aims to know the importance of public health knowledge area in the health sciences degrees, and the perception of the students from the latest grades. Subjects and methods. The study has two parts. The first one describes the educational offer of the subjects from the public health knowledge area. The second one, using a questionnaire, shows the evaluation of those subjects from 361 students of medicine and nursing. Results. Ten of the twelve health sciences degrees taking place in nine Catalan universities, offer subjects of the public health knowledge area, from 3 credits (CTA UB) to 32.5 (community nursing UdL). The students don't have the correct perception of the concept of public health. The evaluation of the nursing students is systematically better than the medicine one. The intentions of the medicine students to work as a public health professional are really poor. Conclusion. Being a first approach, limited to a convenience sample, the findings of the study have to be interpreted carefully, even thought, they don't permit to rule out the hypothesis that the subjects of the public health knowledge area, don't encourage students to think in the future of the professional public health practice as a fact.

Key words. Medicine students. Nursing students. Public health. Universities.

\section{Introducción}

Las definiciones académicas de salud pública son diversas. Las utilizadas con más frecuencia tienen su origen en la reformulación de Terris [1] sobre la proposición de Winslow del año 1920: 'La ciencia y el arte de prevenir las dolencias y las discapacidades, prolongar la vida y fomentar la salud y la eficiencia física y mental, median- a Fundació Universitària del Bages. Manresa, Barcelona. b Institut d'Estudis de la Salut. Barcelona, España.

\section{Correspondencia}

Sra. Núria Obradors Rial.

Fundació Universitària del Bages. Avda. Universitària, 4-6. E-08242 Manresa (Barcelona).

E-mail nobradors@fub.edu

Agradecimientos A todo el profesorado de las universidades por su colaboración en la recogida de los datos y facilitarnos la información necesaria para seguir adelante con el proyecto, como también a los/las decanos/as y directores/as de las escuelas y las facultades por posibilitar el acceso a la información de sus instituciones.

Agradecer también a Olatz Garin su soporte metodológico en todo el trabajo, y al Dr. Josep Roma, al Dr. Fernando G. Benavides y la Sra. Marta Vilanova, su colaboración en la elaboración del cuestionario.

Dar gracias también a la Srta. Luisa Aliste, por su contribución en la parte estadística del trabajo. Finalmente, reconocer la viabilidad de este proyecto gracias al soporte del IES. 
te esfuerzos organizados de la comunidad para sanar el medio ambiente, controlar las enfermedades infecciosas y no infecciosas, así como las lesiones; educar al individuo en los principios de higiene personal, organizar los servicios para el diagnóstico y el tratamiento de las enfermedades y para la rehabilitación, así como desarrollar la maquinaria social que le asegura a cada miembro de la comunidad un nivel de vida adecuado para el mantenimiento de la salud'. Por su parte, Acheson la resume como 'la ciencia y el arte -en el sentido de oficio- de prevenir las enfermedades, prolongar la vida y mejorar la salud mediante el esfuerzo organizado de la sociedad' [2]. De lo indicado se puede deducir que la salud pública tiene dos componentes, uno general, que constituye una infraestructura social, y otro, específicamente sanitario, como componente de los sistemas que desarrollan actividades colectivas de este carácter. En consecuencia, la identificación de la salud pública con los servicios sanitarios financiados públicamente, a pesar de su frecuencia, es equívoca.

Las funciones de la salud pública en la sociedad consisten en valorar las necesidades, desarrollar políticas y garantizar la prestación de servicios [3,4]. En consecuencia, la función de la salud pública en el seno del sistema sanitario debería ser central. Sin embargo, su situación es más bien marginal. En efecto, la media del gasto en servicios de salud pública en los países de la OCDE fue en el año 2005 del 2,9\% del gasto sanitario total, y la cifra correspondiente en España, del 1,3\% [5]. Entre los factores que podrían explicar esta marginalidad [6,7], se ha prestado poca atención a la influencia que pueda tener la formación universitaria. Pese a que asignaturas del área de conocimiento se han incorporado últimamente en los programas de titulaciones diversas, como por ejemplo farmacia o trabajo social, no parece que los estudiantes dispongan de estímulos para considerar su ejercicio profesional futuro en alguna de las actividades de la salud pública [8].

Se ha partido de la hipótesis de que los alumnos de las ciencias de la salud no tienen claro el concepto de salud pública y que la importancia que se le da en los planes de estudio de las titulaciones universitarias es poco relevante, de forma que su eventual influencia para dirigir la vida profesional hacia éste ámbito es muy limitada. La detección de factores determinantes de las valoraciones permite desarrollar intervenciones educativas que aumenten el interés y las expectativas de los estudiantes y contribuyan así al desarrollo de la salud pública.

Los propósitos de este estudio son describir el peso de las asignaturas del área de conocimiento de salud pública en las titulaciones de ciencias de la salud, y explorar de forma preliminar la valoración que la salud pública merece a los estudiantes de las facultades de ciencias de la salud de Cataluña, que han cursado ya las asignaturas de esta área de conocimiento. Y la relación entre esta valoración y el interés y el atractivo que les desvela, básicamente como posible preferencia de ejercicio profesional.

\section{Sujetos y métodos}

En primer lugar, se han examinado las asignaturas del área de conocimiento de salud pública y medicina preventiva [9] existentes en los planes de estudio de las titulaciones de ciencias de la salud de Cataluña [10]. Obtenida la relación de las titulaciones de ciencias de la salud, se ha procedido a identificarlas en los planes de estudio de las titulaciones de cada una de las universidades de Cataluña, para conocer de cada universidad y para cada titulación, cuáles eran las asignaturas del área de conocimiento de salud pública que se impartían y el número de créditos de éstas.

El estudio de campo se ha limitado a obtener las valoraciones de los estudiantes de medicina [11-13] y enfermería [14]. Teniendo en cuenta los personajes históricos de gran relevancia [15], como también las primeras regulaciones de la enseñanza, la medicina es la disciplina con más tradición en salud pública. Así pues, en base a la importancia relativa (peso en el plan de estudios) y la tradición, medicina y enfermería eran las más adecuadas, teniendo en cuenta que por recursos no se podían abarcar la totalidad de las titulaciones de ciencias de la salud.

\section{Selección de la muestra}

Se obtuvo una relación de los decanos/as y los profesores/as titulares de cada asignatura y de cada universidad de medicina y enfermería, con determinación de nombre, dirección, correo electrónico y teléfono de contacto, a partir de las bases de datos de las diferentes universidades 
catalanas, a través del Departamento de Innovación, Universidades y Empresa de la Generalitat de Cataluña [16].

Se mantuvo un primer contacto telefónico, con cada uno de los profesores responsables de las asignaturas de salud pública, para ratificar la información obtenida con anterioridad y presentarles el estudio. A continuación, se les envió un escrito explicativo en relación a los motivos del proyecto y solicitando su participación.

\section{Elaboración del cuestionario}

Mediante la búsqueda bibliográfica inicial no se identificó ningún antecedente que permitiera disponer de un instrumento de medida de las valoraciones de los estudiantes, de forma que se ha elaborado un cuestionario de 21 preguntas, 15 de las cuales son de tipo Likert de cinco ítems de posibilidad de respuesta (de 'muy en desacuerdo' a 'muy de acuerdo'), cinco son de respuesta cerrada y una última abierta. El cuestionario se divide en tres apartados:

- En el primer apartado se solicitan datos sobre el alumno/a: edad, sexo, si están matriculados de último curso y si tienen posibilidades de terminar en el mismo año.

- En el segundo apartado, se explora la percepción que tienen de la salud pública en el sistema sanitario: concepto de salud pública, si consideran que la salud pública debería tener más importancia en el conjunto del sistema sanitario, y si les aporta elementos para el ejercicio profesional; valoración de la formación recibida en salud pública en su titulación, y utilidad de las asignaturas, así como sus expectativas. En referencia al profesorado, si consideran atractivas las clases recibidas, si el nivel de formación es elevado y su prestigio académico alto y, finalmente, su opinión sobre la carga docente, grado de exigencia académico y marginalidad de las asignaturas de salud pública.

- En el tercer y último apartado del cuestionario, se pregunta la posible intención de dedicación a la salud pública, y si han suspendido durante los años de carrera alguna de las asignaturas del área de conocimiento.

Se facilitó el cuestionario a todos los profesores participantes para que expresaran libremente su opinión y poder así incorporar las modificaciones correspondientes. Realizado lo anterior, se redactó definitivamente el cuestionario, solicitando al profesorado que lo administrara al alumnado de último curso, para asegurar que la totalidad de los encuestados habría cursado todas las asignaturas del área de conocimiento de salud pública. Los cuestionarios cumplimentados se devolvieron por correo postal.

\section{Análisis estadístico}

Se han calculado las frecuencias absolutas y relativas según la titulación. Para la comparación de proporciones entre titulaciones se ha usado el test de $\chi^{2}$. Por otro lado, como parte del estudio piloto, se han comprobado algunas de las propiedades métricas del cuestionario: exploración de estructura (utilizando análisis factorial exploratorio, EFE) y análisis de fiabilidad del instrumento a nivel de consistencia interna (grado en que la medida está libre de error aleatorio). Este último paso se ha evaluado utilizando el coeficiente alfa de Cronbach.

\section{Resultados}

\section{Titulaciones de ciencias de la salud}

El Departamento de Innovación, Universidades y Empresa de la Generalitat de Cataluña [10], basándose en la Ley de ordenación de las profesiones sanitarias [17], establece que las titulaciones de ciencias de la salud son: ciencia y tecnología de los alimentos, fisioterapia, logopedia, nutrición humana y dietética, óptica y optometría, terapia ocupacional, farmacia, enfermería, medicina, odontología, podología y veterinaria.

El Real Decreto 1888/1984, de 26 de septiembre [9], determina el catálogo del área de conocimiento de medicina preventiva y salud pública. De este catálogo, y para medicina, se han escogido las asignaturas de epidemiología, demografía sanitaria, estadística, medicina preventiva y salud pública, y para enfermería, las enfermerías comunitarias, la bioestadística y la educación para la salud.

No se han encontrado antecedentes de valoración de las asignaturas de salud pública en las universidades de Cataluña. Las investigaciones 
Tabla I. Créditos dedicados a salud pública en medicina y enfermería.

\begin{tabular}{lcc}
\hline & Mínimo & Máximo \\
\hline Enfermería & & \\
\hline UAB & 4,5 & 28 \\
\hline UB & 7,5 & 18 \\
\hline UPF & 7,5 & 18 \\
\hline UdL & - & 32,5 \\
\hline UdG & 6 & 30 \\
\hline URV & - & 24 \\
\hline URL & 4,5 & 26 \\
\hline UVic & 4,5 & 32 \\
\hline UIC & - & 30 \\
\hline Medicina & 4,5 & 12 \\
\hline UAB & 4,5 & \\
\hline UB & & 32 \\
\hline UdL & & \\
\hline URV & & \\
\hline
\end{tabular}

UAB: Universitat Autònoma de Barcelona; UB: Universitat de Barcelona; UPF: Universitat Pompeu Fabra; UdL: Universitat de Lleida; UdG: Universitat de Girona; URV: Universitat Rovira i Virgili; URL: Universitat Ramon Llull; UVic: Universitat de Vic; UIC: Universitat Internacional de Catalunya.

más próximas al estudio fueron para la titulación de medicina, centradas en la especialidad de medicina de familia, ninguna en salud pública.

\section{Universidades}

En la titulación de ciencia y tecnología de los alimentos impartida en las universidades de Barcelona (UB), Autònoma de Barcelona (UAB), Girona (UdG), Lleida (UdL) y Vic (UVic), se imparte la asignatura de salud pública, con un mínimo de 3 créditos y un máximo de 4,5. En la titulación de fisioterapia, la asignatura de salud pública y legislación sanitaria se imparte en la UAB, UdG, Rovira i Virgili (URV), UVic, Ramon Llull (URL) e Internacional de Catalunya (UIC) con 7 o 7,5 créditos. En los estudios de logopedia (UAB y URL) no hay asignaturas del área de conocimiento de salud pública, ni en los estudios de óptica y optometría impartida en la Politècnica de Catalunya (UPC). En nutrición humana y dietética (UB, UdL, URV, UVic y URL), las asignaturas del área de conocimiento de salud pública oscilan entre 4,5 y 6 créditos. Terapia ocupacional (UVic y UAB) tiene de 4,5 a 6 créditos. Farmacia (UB) ofrece 4,5 y 8 créditos de asignaturas del área de conocimiento de salud pública. De 5 a 8 créditos son los que se imparten en la UB y UIC en odontología. En la UB, en podología, se imparte podología preventiva de 4,5 créditos, mientras que en la UAB es de 6 créditos. Por último, veterinaria (UAB) forma con 4,5 y 6 créditos a su alumnado en asignaturas del área de conocimiento de la salud pública. En la tabla I se observan las universidades que imparten las titulaciones de medicina y enfermería y los créditos que les destinan.

\section{Participación en el estudio}

La población de estudio ha sido el alumnado matriculado de último curso, en las titulaciones de enfermería, ( $3^{\text {er. }}$ curso) y de medicina ( $6^{\circ}$ curso) de Cataluña, pertenecientes a 15 escuelas universitarias de enfermería y cuatro facultades de medicina. De éstas participaron definitivamente tres universidades de medicina y ocho escuelas de enfermería. El número total de estudiantes que respondieron a los cuestionarios fue de 361 .

\section{Resultados de los cuestionarios}

\section{Descripción de la muestra}

En total se han recogido 135 cuestionarios cumplimentados de alumnos de enfermería y 226 de medicina. En la tabla II se observa que la media de edad de los estudiantes de enfermería es superior y que las mujeres predominan en los últimos cursos de ambas titulaciones, con mayor proporción en enfermería.

Casi la totalidad del alumnado de enfermería estaba matriculado de último curso, mientras que en medicina eran las tres cuartas partes. El 
$70 \%$ de los estudiantes de medicina y el $90 \%$ de los de enfermería estaban en disposición de terminar la carrera aquel curso. La proporción de suspendidos en alguna asignatura de salud pública durante la carrera fue similar entre las dos titulaciones, próxima al $40 \%$.

\section{Percepción del alumnado}

Como se muestra en la tabla III, el 75\% del alumnado de enfermería identifica la salud pública con el conjunto de servicios sanitarios financiados públicamente, proporción que es del $70 \%$ entre los alumnos de medicina.

La inmensa mayoría de los estudiantes de ambas titulaciones están de acuerdo o muy de acuerdo con la afirmación de que la salud pública debería tener mayor importancia dentro del sistema sanitario, aunque el $7 \%$ de los estudiantes de enfermería y el $12 \%$ de los de medicina no lo comparten.

Un $4 \%$ de los estudiantes de medicina manifiesta su desacuerdo con la afirmación de que la salud pública aporta elementos positivos para el ejercicio profesional.

\section{Valoración de la formación en salud pública}

En la tabla IV se pueden observar las valoraciones de los estudiantes de medicina y enfermería. La valoración que les merece la formación en salud pública a los estudiantes de enfermería es sistemáticamente mejor que la percepción de los estudiantes de medicina.

Mientras que para el $22 \%$ de los estudiantes de enfermería la formación ha sido muy útil, sólo lo ha sido para el $6 \%$ de los estudiantes de medicina. Las expectativas han sido superadas para uno de cada tres estudiantes de enfermería, pero no para más de la mitad de los de medicina, un $13 \%$ de los cuales las valoran negativamente. Las clases han sido muy atractivas para el $18 \%$ de los estudiantes de enfermería, pero sólo para el 3\% de los de medicina. Uno de cada cinco estudiantes de medicina considera que los profesores no hacen atractivas las clases. Sólo el 11\% de los estudiantes de enfermería y menos del 3\% de los de medicina consideran que los profesores de salud pública son los de mayor prestigio académico. La mitad de los estudiantes de medicina no cree que sean necesarias más horas de las asignaturas del área de conocimiento. Los estudiantes de enfermería tienen una percepción más acusada del grado de exigencia de las asignaturas de salud
Tabla II. Características de los estudiantes de enfermería y medicina ${ }^{a}$.

\begin{tabular}{lcc}
\hline & $\begin{array}{c}\text { Enfermería } \\
(n=135)\end{array}$ & $\begin{array}{c}\text { Medicina } \\
(n=226)\end{array}$ \\
\hline Edad (años) & $24,7 \pm 5,62$ & $23,9 \pm 2,08$ \\
\hline Media \pm DE & $20-46$ & $21-44$ \\
\hline Rango & $109(80,7 \%)$ & $169(74,8 \%)$ \\
\hline Sexo & $26(19,3 \%)$ & $57(25,2 \%)$ \\
\hline Mujer & $134(99,3 \%)$ & $165(73,0 \%)$ \\
\hline Hombre & $0(0 \%)$ & $61(27,0 \%)$ \\
\hline Matriculado ${ }^{b}$ & $119(88,1 \%)$ & $154(68,1 \%)$ \\
\hline Sí & $15(11,1 \%)$ & $70(31,1 \%)$ \\
\hline No & & \\
\hline Posibilidades de terminarc & \\
\hline Sí & & \\
\hline No & & \\
\hline
\end{tabular}

Suspenso en salud pública durante la carrera ${ }^{\mathrm{d}}$

\begin{tabular}{lcc}
\hline Sí & $51(37,8 \%)$ & $87(38,5 \%)$ \\
\hline No & $39(28,9 \%)$ & $134(59,3 \%)$ \\
\hline Convocatoria máxima & & \\
\hline Segunda & $35(68,6 \%)$ & $60(69,0 \%)$ \\
\hline Tercera & $7(13,7 \%)$ & $18(20,7 \%)$ \\
\hline Cuarta & $4(7,8 \%)$ & $6(6,9 \%)$ \\
\hline Quinta & $3(5,8 \%)$ & $1(1,1 \%)$ \\
\hline Sexta & $1(2,0 \%)$ & $2(2,3 \%)$ \\
\hline
\end{tabular}

DE: desviación estándar. ${ }^{a}$ Valores perdidos son el residuo de los valores totales; ${ }^{b}$ Alumnado que estaba matriculado de último curso; cAlumnado que tenía posibilidades de diplomarse o licenciarse ese curso; 'Alumnado que ha suspendido alguna de las asignaturas del área de conocimiento de la salud pública durante los años de la carrera; ${ }^{e}$ Quienes habían respondido afirmativamente a la pregunta anterior debían responder a qué convocatoria máxima habían llegado (enfermería: $n=51$; medicina: $n=87$ ).

pública, pero una cuarta parte consideran que son marginales, una valoración que en el caso de medicina sobrepasa el 35\%. 
Tabla III. Percepción del alumnado de salud pública (SP) en el sistema sanitario según las expectativas de ejercicio profesional y la titulación ${ }^{\text {a. }}$

\begin{tabular}{|c|c|c|c|c|c|c|c|c|c|c|c|c|}
\hline & \multicolumn{6}{|c|}{ Enfermería } & \multicolumn{6}{|c|}{ Medicina } \\
\hline & \multicolumn{2}{|c|}{ Total $^{\mathrm{b}}$} & \multicolumn{2}{|c|}{ Síc } & \multicolumn{2}{|c|}{$\mathrm{No}^{\mathrm{d}}$} & \multicolumn{2}{|c|}{ Total $^{\mathrm{b}}$} & \multicolumn{2}{|c|}{ Síc } & \multicolumn{2}{|c|}{$\mathrm{No}^{d}$} \\
\hline & $n$ & $\%$ & $n$ & $\%$ & $n$ & $\%$ & $n$ & $\%$ & $n$ & $\%$ & $n$ & $\%$ \\
\hline \multicolumn{13}{|l|}{ Concepto de SPg } \\
\hline Muy de acuerdo & 28 & 20,7 & 15 & 20,5 & 11 & 20,4 & 31 & 13,7 & 1 & 12,5 & 29 & 13,8 \\
\hline De acuerdo & 75 & 55,6 & 41 & 56,2 & 30 & 55,6 & 126 & 55,8 & 6 & 75,0 & 118 & 56,2 \\
\hline Ni acuerdo ni desacuerdo & 19 & 14,1 & 11 & 15,1 & 7 & 13,0 & 21 & 9,3 & - & - & 19 & 9,0 \\
\hline En desacuerdo & 8 & 5,9 & 5 & 6,8 & 3 & 5,6 & 32 & 14,2 & 1 & 12,5 & 31 & 14,8 \\
\hline Muy en desacuerdo & 4 & 3,0 & 1 & 1,4 & 3 & 5,6 & 13 & 5,8 & - & - & 13 & 6,2 \\
\hline$p^{e}$ & \multicolumn{6}{|c|}{0,753} & \multicolumn{6}{|c|}{0,784} \\
\hline \multicolumn{13}{|l|}{$\begin{array}{l}\text { Importancia de la SP } \\
\text { en el sistema sanitario }\end{array}$} \\
\hline Muy de acuerdo & 56 & 41,5 & 35 & 47,9 & 19 & 34,5 & 85 & 37,6 & 5 & 62,5 & 76 & 36,0 \\
\hline De acuerdo & 70 & 51,9 & 36 & 49,3 & 29 & 52,7 & 114 & 50,4 & 3 & 37,5 & 108 & 51,2 \\
\hline Ni acuerdo ni desacuerdo & 8 & 5,9 & 2 & 2,7 & 6 & 10,9 & 26 & 11,5 & - & - & 26 & 12,3 \\
\hline En desacuerdo & 1 & 0,7 & - & - & 1 & 1,8 & 1 & 0,4 & - & - & 1 & 0,5 \\
\hline Muy en desacuerdo & - & - & - & - & - & - & - & - & - & - & - & - \\
\hline$p^{e}$ & \multicolumn{6}{|c|}{0,108} & \multicolumn{6}{|c|}{0,428} \\
\hline \multicolumn{13}{|l|}{$\begin{array}{l}\text { Visión de la SP en el } \\
\text { ejercicio profesional }^{i}\end{array}$} \\
\hline Muy de acuerdo & 37 & 27,4 & 27 & 37,0 & 9 & 16,7 & 82 & 36,3 & 5 & 62,5 & 73 & 35,8 \\
\hline De acuerdo & 82 & 60,7 & 39 & 53,4 & 37 & 68,5 & 117 & 51,8 & 3 & 37,5 & 112 & 54,9 \\
\hline Ni acuerdo ni desacuerdo & 15 & 11,1 & 7 & 9,6 & 8 & 14,8 & 18 & 8,0 & - & - & 17 & 8,3 \\
\hline En desacuerdo & - & - & - & - & - & - & 2 & 0,9 & - & - & 2 & 1,0 \\
\hline Muy en desacuerdo & - & - & - & - & - & - & 7 & 3,1 & - & - & - & - \\
\hline$p^{e}$ & \multicolumn{6}{|c|}{$0,040^{f}$} & \multicolumn{6}{|c|}{0,446} \\
\hline Alumnos & 135 & 100,0 & 73 & 100,0 & 55 & 100,0 & 226 & 100,0 & 8 & 100,0 & 211 & 100,0 \\
\hline
\end{tabular}

\section{Expectativas de ejercicio profesional}

Más de la mitad de los estudiantes de enfermería no descartan dedicarse profesionalmente a la salud pública o a la salud comunitaria a corto o largo plazo, mientras que sólo ocho de los estudiantes de medicina $(3,5 \%)$ se declaran dispuestos a escoger la especialidad en el próximo MIR (Tabla V). 
Tabla IV. Valoración de la formación en salud pública (SP) según las expectativas de ejercicio profesional y la titulación a.

\begin{tabular}{|c|c|c|c|c|c|c|c|c|c|c|c|c|c|}
\hline & & \multicolumn{6}{|c|}{ Enfermería } & \multicolumn{6}{|c|}{ Medicina } \\
\hline & & \multicolumn{2}{|c|}{ Total $^{\mathrm{b}}$} & \multicolumn{2}{|c|}{ Síc } & \multicolumn{2}{|c|}{$\mathrm{No}^{d}$} & \multicolumn{2}{|c|}{ Total $^{\mathrm{b}}$} & \multicolumn{2}{|c|}{ Síc } & \multicolumn{2}{|c|}{$\mathrm{No}^{d}$} \\
\hline & & $n$ & $\%$ & $n$ & $\%$ & $n$ & $\%$ & $n$ & $\%$ & $n$ & $\%$ & $n$ & $\%$ \\
\hline \multirow[t]{6}{*}{ Asignaturas útiles ${ }^{f}$} & Muy de acuerdo & 27 & 20,0 & 17 & 23,3 & 9 & 16,7 & 13 & 5,8 & 2 & 25,0 & 11 & 5,2 \\
\hline & De acuerdo & 80 & 59,3 & 45 & 61,6 & 32 & 59,3 & 111 & 49,1 & 5 & 62,5 & 103 & 48,8 \\
\hline & Ni acuerdo ni desacuerdo & 24 & 17,8 & 9 & 12,3 & 12 & 22,2 & 84 & 37,2 & 1 & 12,5 & 79 & 37,4 \\
\hline & En desacuerdo & 3 & 2,2 & 2 & 2,7 & 1 & 1,9 & 15 & 6,6 & - & - & 15 & 7,1 \\
\hline & Muy en desacuerdo & & 0 & - & - & - & - & 3 & 1,3 & - & - & 3 & 1,4 \\
\hline & $p^{\mathrm{e}}$ & \multicolumn{6}{|c|}{0,451} & \multicolumn{6}{|c|}{0,118} \\
\hline \multirow{6}{*}{$\begin{array}{l}\text { Superadas las } \\
\text { expectativas }\end{array}$} & Muy de acuerdo & 34 & 25,2 & 18 & 25,7 & 14 & 25,5 & 14 & 6,2 & 1 & 16,7 & 13 & 7,0 \\
\hline & De acuerdo & 65 & 48,1 & 41 & 58,6 & 23 & 41,8 & 72 & 31,9 & 2 & 33,3 & 68 & 36,4 \\
\hline & Ni acuerdo ni desacuerdo & 30 & 22,2 & 10 & 14,3 & 17 & 30,9 & 83 & 36,7 & 2 & 33,3 & 80 & 42,8 \\
\hline & En desacuerdo & 2 & 1,5 & 1 & 1,4 & 1 & 1,8 & 26 & 11,5 & 1 & 16,7 & 23 & 12,3 \\
\hline & Muy en desacuerdo & - & - & - & - & - & - & 3 & 1,3 & - & - & 3 & 1,6 \\
\hline & $p^{e}$ & \multicolumn{6}{|c|}{0,451} & \multicolumn{6}{|c|}{0,898} \\
\hline \multirow[t]{6}{*}{ Clases atractivas ${ }^{h}$} & Muy de acuerdo & 22 & 16,3 & 10 & 13,7 & 11 & 20,0 & 7 & 3,1 & 1 & 12,5 & 6 & 2,8 \\
\hline & De acuerdo & 79 & 58,5 & 43 & 58,9 & 32 & 58,2 & 60 & 26,5 & 2 & 25,0 & 57 & 27,0 \\
\hline & Ni acuerdo ni desacuerdo & 28 & 20,7 & 16 & 21,9 & 11 & 20,0 & 114 & 50,4 & 4 & 50,0 & 106 & 50,2 \\
\hline & En desacuerdo & 5 & 3,7 & 4 & 5,5 & 1 & 1,8 & 38 & 16,8 & 1 & 12,5 & 35 & 16,6 \\
\hline & Muy en desacuerdo & - & - & - & - & - & - & 7 & 3,1 & - & - & 7 & 3,3 \\
\hline & $p^{e}$ & \multicolumn{6}{|c|}{0,595} & \multicolumn{6}{|c|}{0,626} \\
\hline
\end{tabular}

Valoración de la formación en salud pública según las expectativas de ejercicio profesional y la titulación

La visión que aporta la salud pública para el ejercicio profesional, según las expectativas del propio ejercicio, ha resultado estadísticamente significativa entre el alumnado de enfermería $(p$ $<0,040$ ), es decir, el alumnado que tiene intención de dedicarse a la salud pública o enfermería comunitaria, a corto o largo plazo, cree que la visión de la salud pública les aporta elementos para el ejercicio profesional de la enfermería, mientras que en medicina no se ha observado esta asociación (Tabla IV).
Valoración de la formación en salud pública según las expectativas de ejercicio profesional No se han observado asociaciones estadísticamente significativas en ninguna de las dos titulaciones. No hay asociación entre las expectativas del ejercicio profesional y su opinión sobre la utilidad de las asignaturas, como tampoco si la formación recibida había superado sus expectativas iniciales, o si los profesores habían hecho las clases atractivas, tampoco el nivel de formación del profesorado o el prestigio académico de los profesores, el número de horas inferior a las necesarias, el grado de exigencia de las asignaturas como uno de los más altos de la carrera, ni si las asignaturas 
Tabla IV. Valoración de la formación en salud pública (SP) según las expectativas de ejercicio profesional y la titulación a (cont.)

\begin{tabular}{|c|c|c|c|c|c|c|c|c|c|c|c|c|c|}
\hline & & \multicolumn{6}{|c|}{ Enfermería } & \multicolumn{6}{|c|}{ Medicina } \\
\hline & & \multicolumn{2}{|c|}{ Total $^{b}$} & \multicolumn{2}{|c|}{ Síc } & \multicolumn{2}{|c|}{$\mathrm{No}^{d}$} & \multicolumn{2}{|c|}{ Total $^{b}$} & \multicolumn{2}{|c|}{ Síc } & \multicolumn{2}{|c|}{$\mathrm{No}^{d}$} \\
\hline & & $n$ & $\%$ & $n$ & $\%$ & $n$ & $\%$ & $n$ & $\%$ & $n$ & $\%$ & $n$ & $\%$ \\
\hline \multirow[t]{6}{*}{ Nivel del profesorado ${ }^{i}$} & Muy de acuerdo & 70 & 51,9 & 35 & 47,9 & 33 & 60,0 & 47 & 20,8 & 2 & 25,0 & 44 & 20,9 \\
\hline & De acuerdo & 52 & 38,5 & 32 & 43,8 & 16 & 29,1 & 138 & 61,1 & 5 & 62,5 & 128 & 60,7 \\
\hline & Ni acuerdo ni desacuerdo & 12 & 8,9 & 6 & 8,2 & 6 & 10,9 & 36 & 15,9 & 1 & 12,5 & 35 & 16,6 \\
\hline & En desacuerdo & - & - & - & - & - & - & 4 & 1,8 & - & - & 4 & 1,9 \\
\hline & Muy en desacuerdo & - & - & - & - & - & - & & 0 & - & - & - & - \\
\hline & $p^{\mathrm{e}}$ & \multicolumn{6}{|c|}{0,232} & \multicolumn{6}{|c|}{0,961} \\
\hline \multirow[t]{6}{*}{ Prestigio académico ${ }^{j}$} & Muy de acuerdo & 15 & 11,1 & 10 & 13,7 & 5 & 9,3 & 6 & 2,7 & 1 & 12,5 & 4 & 1,9 \\
\hline & De acuerdo & 35 & 25,9 & 20 & 27,4 & 14 & 25,9 & 31 & 13,7 & 2 & 25,0 & 28 & 13,4 \\
\hline & Ni acuerdo ni desacuerdo & 68 & 50,4 & 36 & 49,3 & 27 & 50,0 & 106 & 46,9 & 2 & 25,0 & 102 & 48,8 \\
\hline & En desacuerdo & 14 & 10,4 & 6 & 8,2 & 8 & 14,8 & 69 & 30,5 & 3 & 37,5 & 64 & 30,6 \\
\hline & Muy en desacuerdo & 1 & 0,7 & 1 & 1,4 & - & - & 11 & 4,9 & - & - & 11 & 5,3 \\
\hline & $p^{\mathrm{e}}$ & \multicolumn{6}{|c|}{0,643} & \multicolumn{6}{|c|}{0,203} \\
\hline \multirow[t]{6}{*}{ N. ${ }^{\circ}$ de horas inferior ${ }^{k}$} & Muy de acuerdo & 18 & 13,3 & 14 & 19,2 & 3 & 5,8 & 6 & 2,7 & - & - & 5 & 2,4 \\
\hline & De acuerdo & 25 & 18,5 & 16 & 21,9 & 9 & 17,3 & 30 & 13,3 & 3 & 37,5 & 26 & 12,6 \\
\hline & Ni acuerdo ni desacuerdo & 64 & 47,4 & 32 & 43,8 & 30 & 57,7 & 79 & 35,0 & 4 & 50,0 & 73 & 35,3 \\
\hline & En desacuerdo & 19 & 14,1 & 9 & 12,3 & 8 & 15,4 & 89 & 39,4 & 1 & 12,5 & 86 & 41,5 \\
\hline & Muy en desacuerdo & 5 & 3,7 & 2 & 2,7 & 2 & 3,8 & 17 & 7,5 & - & - & 17 & 8,2 \\
\hline & $p^{e}$ & \multicolumn{6}{|c|}{0,212} & \multicolumn{6}{|c|}{0,166} \\
\hline \multirow[t]{6}{*}{ Grado de exigencia' } & Muy de acuerdo & 10 & 7,4 & 7 & 9,6 & 2 & 3,7 & 8 & 3,5 & 1 & 12,5 & 7 & 3,3 \\
\hline & De acuerdo & 53 & 39,3 & 31 & 42,5 & 20 & 37,0 & 37 & 16,4 & - & - & 35 & 16,7 \\
\hline & Ni acuerdo ni desacuerdo & 41 & 30,4 & 22 & 30,1 & 18 & 33,3 & 90 & 39,8 & 4 & 50,0 & 83 & 39,7 \\
\hline & En desacuerdo & 28 & 20,7 & 13 & 17,8 & 13 & 24,1 & 77 & 34,1 & 2 & 25,0 & 75 & 35,9 \\
\hline & Muy en desacuerdo & 1 & 0,7 & - & - & 1 & 1,9 & 10 & 4,4 & 1 & 12,5 & 9 & 4,3 \\
\hline & $p^{\mathrm{e}}$ & \multicolumn{6}{|c|}{0,435} & \multicolumn{6}{|c|}{0,323} \\
\hline \multirow{6}{*}{$\begin{array}{l}\text { Asignaturas } \\
\text { marginales }^{\mathrm{mw}}\end{array}$} & Muy de acuerdo & 9 & 6,7 & 8 & 11,1 & 1 & 1,9 & 5 & 2,2 & - & - & 5 & 2,4 \\
\hline & De acuerdo & 21 & 15,6 & 11 & 15,3 & 10 & 18,5 & 75 & 33,2 & 4 & 50,0 & 70 & 33,8 \\
\hline & Ni acuerdo ni desacuerdo & 46 & 34,1 & 27 & 37,5 & 18 & 33,3 & 74 & 32,7 & 1 & 12,5 & 71 & 34,3 \\
\hline & En desacuerdo & 44 & 32,6 & 22 & 30,6 & 20 & 37,0 & 56 & 24,8 & 3 & 37,5 & 52 & 25,1 \\
\hline & Muy en desacuerdo & 10 & 7,4 & 4 & 5,6 & 5 & 9,3 & 10 & 4,4 & - & - & 9 & 4,3 \\
\hline & $p^{\mathrm{e}}$ & \multicolumn{6}{|c|}{0,284} & \multicolumn{6}{|c|}{0,612} \\
\hline \multicolumn{2}{|l|}{ Alumnos } & 135 & 100,0 & 73 & 100,0 & 55 & 100,0 & 226 & 100,0 & 8 & 100,0 & 211 & 100,0 \\
\hline \multicolumn{14}{|c|}{ 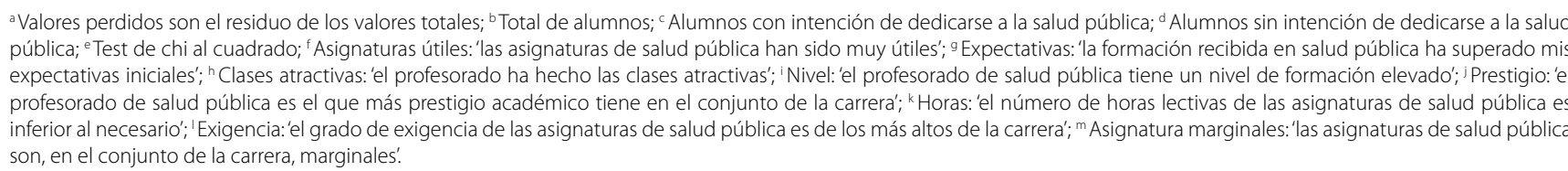 } \\
\hline
\end{tabular}


de salud pública las consideraban marginales en el conjunto de la carrera (Tabla IV).

\section{Evaluación de las propiedades métricas del cuestionario}

Se han transformado las opciones de respuesta de dos de los ítems, los cuales estaban formados por entre tres y seis subítems (en función de la universidad), haciendo una media. El cálculo de la puntuación global del instrumento se ha realizado por sumatorio de sus opciones de respuesta. Algunos de los ítems presentaban hasta un $4,4 \%$ de valores perdidos. Según los resultados del EFE, el cuestionario presenta cuatro factores. Aun así, el primer factor ya explica el $70 \%$ de la varianza total. Para la puntuación global del instrumento, el alfa de Cronbach fue de 0,79, superando así el estándar propuesto para comparaciones de grupos.

\section{Discusión}

Esta investigación es la primera aproximación dirigida a explorar el peso de las asignaturas del área de conocimiento de salud pública en las titulaciones universitarias de ciencias de la salud. Para conocer la opinión de los estudiantes se llevó a cabo una prueba piloto que tiene limitaciones, entre las que destaca que la mayoría de los cuestionarios se administraron aprovechando el día del examen de una de las asignaturas de salud pública, dado que durante el último curso de la carrera se realizan muchas prácticas asistenciales y el alumnado tan sólo acude a la universidad para los exámenes. Así, hace falta tener en cuenta la posible interferencia sobre los datos, pese a que el cuestionario fuera anónimo. Además, no todos los alumnos de último curso de las titulaciones respondieron al cuestionario, como tampoco participaron todas las universidades a las que se invitó; por tanto, la muestra no es representativa de las universidades de Cataluña de medicina y enfermería.

Sin embargo, llama la atención la confusión sobre lo qué es la salud pública, identificada erróneamente con el conjunto de los servicios sanitarios financiados públicamente. Sólo uno de cada diez estudiantes de enfermería (9\%) y uno de cada cinco (20\%) de medicina manifestaron su desacuerdo con la afirmación, lo que sugiere que
Tabla V. Futuro profesional en enfermería y medicina ${ }^{a}$.

\begin{tabular}{lcc}
\hline & $\begin{array}{c}\text { Enfermería } \\
(n=135)\end{array}$ & $\begin{array}{c}\text { Medicinac } \\
(n=226)\end{array}$ \\
\hline Sí & $73(54,1 \%)$ & $8(3,5 \%)$ \\
\hline No & $55(40,7 \%)$ & $211(93,4 \%)$ \\
\hline
\end{tabular}

a Valores perdidos son el residuo de los valores totales; ${ }^{\text {b }}$ La formulación de la pregunta en enfermería ha sido: '¿tienes intención de dedicarte a enfermería comunitaria o salud pública a corto o largo plazo?'; ' 'La formulación de la pregunta en medicina ha sido:'¿escogerás la especialidad de medicina preventiva y salud pública en el próximo MIR?'

los conocimientos aportados no son adecuados o que, al menos, no llegan a los estudiantes.

La valoración de la formación recibida es diferente según la titulación; aun cuando no puede atribuirse a la actividad de los profesores, atendida la naturaleza de la información, es suficientemente grande como para requerir una profundización. Es posible también que la influencia de aspectos más relacionados con la salud comunitaria en los estudios de enfermería tenga un papel relevante.

Si seleccionamos la valoración más alta posible de cada uno de los aspectos explorados, los estudiantes de enfermería son los que con más frecuencia las escogen de forma sistemática, incluso cuando la pregunta tiene que ver con la marginalidad de las asignaturas. Esta característica de las respuestas hace pensar en un patrón propio de respuesta asociado a enfermería que puede distorsionar algunas comparaciones. No obstante, también parece verosímil que la valoración de la marginalidad de las asignaturas por parte de enfermería se relacione con la confusión sobre la naturaleza de la salud pública y la salud comunitaria.

En cuanto a los estudiantes de medicina, sus respuestas muestran un patrón consistente, que sugiere que la salud pública les interesa poco, está sobredimensionada para sus preferencias, que los profesores no la hacen especialmente interesante y que no son los que disfrutan de mayor prestigio docente.

Para los estudiantes de medicina, la salud pública no es una salida profesional atractiva, aun cuando las peculiaridades personales puedan explicar que el número de personas que no des- 
cartan hacer la especialidad médica supere a los que prefieran la medicina familiar y comunitaria, una elección para la cual los estímulos formativos son más escasos todavía. En cuanto a las expectativas de los estudiantes de enfermería, la confusión entre salud pública y salud comunitaria puede explicar la aparente preferencia.

En la comparación de proporciones se ha observado asociación entre los estudiantes de enfermería que quieren dedicarse a la salud pública o enfermería comunitaria, a corto o largo plazo, y la visión que les aporta elementos para su ejercicio profesional. Es posible también, como ya se ha mencionado, que la influencia de aspectos más relacionados con la salud comunitaria tenga un papel relevante. No se han encontrado asociaciones con los estudiantes de medicina en ninguna de las variables, teniendo en cuenta, sin embargo, que el número de estudiantes que afirmaba escoger medicina preventiva y salud pública como especialidad en el próximo MIR ha sido tan sólo del 3,5\%, que implica una baja potencia estadística para poder encontrar asociaciones.

Teniendo en cuenta que es una prueba piloto, creemos que los resultados del estudio no descartan las hipótesis iniciales, de forma que conviene ampliar el estudio con una participación más elevada y considerando el resto de titulaciones de ciencias de la salud, pudiendo así analizar las necesidades formativas de los estudiantes con el objetivo final de conseguir la equiparación entre las responsabilidades que se atribuyen a la salud pública dentro del sistema y los recursos que se le destinan.

\section{Bibliografía}

1. Terris M. Tendencias actuales en la salud pública. In Organización Panamericana de la Salud, ed. La crisis de la salud pública: reflexiones para el debate. Washington DC: OPS; 1992. p. 185-204.

2. Acheson D. Public health in England: the report of the Committee of Inquiry into the Future Development of the Public Health Function. Cmnd 289. London: HMSO; 1998.

3. De la Puente ML, Manzanera R. Grupo de Trabajo de Salud Pública en Cataluña: propuesta marco de reorganización de la salud pública en Cataluña. Gac Sanit 2001; 15 (Supl 4): 55-68.
4. Benavides F, Moya C, Segura A, De la Puente ML, Porta $\mathrm{M}$, Amela C. Las competencias profesionales en salud pública. Gac Sanit 2006; 20: 239-43.

5. Rodríguez M, Stoyanova A. Els comptes de la salut pública a Catalunya. Descripció, anàlisi i metodologia de càlcul de la despesa en salut pública a Catalunya, 2005. Barcelona: Direcció General de Salut Pública. Departament de Salut de la Generalitat; 2005.

6. Navarro V. L'estat del benestar a Catalunya. Barcelona: Diputació de Barcelona, Oficina de la Xarxa de Barcelona Municipis de Qualitat; 2003.

7. Álvarez-Dardet C, Peiró S, eds. La salud pública ante los desafíos de un nuevo siglo. Informe SESPAS 2000. Granada: Escuela Andaluza de Salud Pública; 2000.

8. Segura A. Salud pública: nueva sección de Educación Médica. Educ Med 2007; 10: 3-6.

9. Real Decreto $1888 / 1984$, de 26 de septiembre, por el que se regulan los concursos para la provisión de plazas de los cuerpos docentes universitarios. BOE n. ${ }^{\circ} 257$, de 26 de octubre de 1984.

10. Generalitat de Catalunya. Què i per què estudiar a les universitats catalanes? URL: http://www10.gencat.cat/ cur_wrcec/AppJava/visible/estudis/estudisPerAmbit.do; jsessionid $=\mathrm{c} 0 \mathrm{a} 833141 \mathrm{f} 45220 \mathrm{ed} 925 \mathrm{cb} 5345529 \mathrm{ce} 9 \mathrm{c} 23 \mathrm{c} 33$ d1d8df?method=list\&tipus=M. [13.06.2009].

11. Ley de 20 de julio de 1955 sobre enseñanza, título y ejercicio de las especialidades médicas. BOE n. ${ }^{\circ}$ 202, de 21 de julio de 1955. p. 4440-2.

12. Orden de 10 de octubre de 1962 por la que se dan normas para la concesión de los títulos de médicos especialistas. BOE n. ${ }^{\circ}$ 272, de 13 de noviembre de 1962.

13. Real Decreto $1417 / 1990$, de 26 de octubre, por el que se establece el título universitario oficial de Licenciado en Medicina y las directrices generales propias de los planes de estudios conducentes a la obtención de aquél. BOE n. ${ }^{\circ}$. 278, de 20 de noviembre de 1990.

14. Real Decreto $1466 / 1990$, de 26 de octubre, por el que se establece el titulo universitario oficial de Diplomado en Enfermería y las directrices generales propias de los planes de estudios conducentes a la obtención de aquél. BOE n. ${ }^{\circ} 278$, de 20 de noviembre de 1990.

15. Cerda J, Valdivia G. John Snow, the cholera epidemic and the foundation of modern epidemiology. Rev Chil Infectol 2007; 24: 331-4.

16. Generalitat de Catalunya. Llista d'universitats. Departament d'Innovació, Universitats i Empresa. URL: http:// www.gencat.cat/diue/ambits/ur/universitats/sistema/ universitat_catalanes/llista/index.html. [13.06.2009].

17. Ley $44 / 2003$, de 21 de noviembre, de ordenación de las profesiones sanitarias. BOE $n .^{\circ} 280$, de 22 de noviembre de 2003. 